\title{
Separación selectiva de hierro y cromo de las lejías agotadas del decapado de acero inoxidable
}

\author{
J.L. Gálvez*, J. Dufour*, C. Negro*y F. López-Mateos*
}

Resumen

\begin{abstract}
Los baños ácidos agotados del decapado de acero inoxidable son disoluciones muy complejas debido al alto contenido de metales y ácidos $\left(\mathrm{HNO}_{3}\right.$ y $\left.\mathrm{HF}\right)$, por lo que constituyen un grave problema medioambiental. Existen tratamientos comerciales para la recuperación del ácido (libre o complejado) que se basan en técnicas de retardo ácido, extracción con disolventes, evaporación o membranas. En estos procesos el contenido metálico es precipitado y tratado como un residuo. El grupo de investigación formado por los autores del presente trabajo ha desarrollado un procedimiento que permite el aprovechamiento de dichos metales mediante su recuperación selectiva con una técnica de precipitación modificada inducida por fluoruro libre. Se consigue la precipitación de hierro (Fe) y cromo $(\mathrm{Cr})$ como pentafluoruros $(\mathrm{pH} 3-4,5)$ dejando el níquel en disolución. Posteriormente, los fluoruros complejos son hidrolizados con álcali dando lugar a hidróxidos de hierro y cromo, mientras que el fluoruro es redisuelto.
\end{abstract}

Palabras clave Acero inoxidable. Decapado. Precipitación selectiva. Hierro. Cromo. Níquel.

\section{Selective separation of iron and chromium from stainless steel spent pickling}

\begin{abstract}
Stainless steel spent pickling baths are very complex solutions of metals and acids $\left(\mathrm{HNO}_{3}\right.$ and HF) and are a very important environmental concern. Several processes have been developed for acid recovery (free and bounded acid) with techniques like acid retardation, solvent extraction, evaporation and dialysis diffusion. In these processes, metallic content is precipitated and treated for its disposal. We have developed a process that permits the separation of metals by means of a selective precipitation, induced by adding free fluoride. Iron $(\mathrm{Fe})$ and chromium $(\mathrm{Cr})$ precipitate as pentafluorides and nitrogennickel $(\mathrm{Ni})$ remains in solution. After this stage, complex fluorides can be hydrolized with alkali to give iron and chromium hydroxides, releasing fluoride in solution.
\end{abstract}

Keywords Stainless steel. Pickling. Selective precipitation. Iron. Chromium. Nickel.

\section{INTRODUCCIÓN}

En el proceso de decapado de acero inoxidable, el baño ácido debe ser retirado cuando se alcanzan concentraciones metálicas de, aproximadamente, un $5 \%$. Este baño debe ser reemplazado por ácido fresco, dando lugar a un residuo de composición muy compleja (Tabla I).

Como se observa en la tabla I, la diferencia entre ácido fluorhídrico y fluoruro total en disolución difieren considerablemente, debido a la formación de complejos metálicos del tipo $\mathrm{FeF}_{2}{ }^{-}$ó $\mathrm{CrF}_{2}{ }^{-}$. La formación de estos complejos condiciona de manera importante la química de este tipo de disoluciones ${ }^{[1]}$.
Tabla I. Composición media de los baños ácidos agotados procedentes del decapado

Table I. Mean composition of spent pickling bath

\begin{tabular}{cc}
\hline Componente & Composición (g/l) \\
\hline $\mathrm{Fe}$ & $35-45$ \\
$\mathrm{Cr}$ & $8-11$ \\
$\mathrm{Ni}$ & $3-6$ \\
$\mathrm{Mo}$ & 1 \\
$\mathrm{HNO}_{3}$ & $120-140$ \\
$\mathrm{HF}$ & $20-30$ \\
$\mathrm{NO}_{3}{ }^{-}$total & $130-150$ \\
$\mathrm{~F}^{-}$total & $60-80$ \\
\hline
\end{tabular}

(*) Dpto. de Ingeniería Química, Universidad Complutense de Madrid, Avda. Complutense s/n, 28040 Madrid. 
Separación selectiva de hierro y cromo de las lejías agotadas del decapado de acero inoxidable

J.L. GÁlVEz, J. Dufour, C. NEGRO y F. LÓPEZ-Mateos

Los procesos de tratamiento de estos baños agotados se centran en la recuperación del contenido ácido, ya sea libre o total, mediante técnicas como el retardo ácido ${ }^{[2]}$, evaporación con ácido sulfúri$\mathrm{Co}^{[3]}$ y técnicas de membrana ${ }^{[4}$ y 5$]$. Sin embargo, la recuperación de los metales se aborda como un problema secundario y, en la mayoría de los casos, las corrientes residuales de estos procesos recurren a la precipitación o neutralización y traslado de la torta metálica a vertedero. En el proceso Pyromars $^{[6]}$, se realiza una pirohidrólisis a $300-400{ }^{\circ} \mathrm{C}$ de la lejía obteniendo unos óxidos metálicos que pueden reaprovecharse en el proceso siderúrgico. En la industria hidrometalúrgica se emplean numerosas técnicas de extracción para la recuperación de metales ${ }^{[7 \text { y } 8]}$. En el caso de las lejías de decapado ha sido aplicada con éxito en el proceso Kawasa$k i^{[9]}$, recuperando el $95 \%$ del contenido en hierro mediante extracción con D2HEPA. No se han desarrollado procesos de precipitación enfocados a la recuperación de los metales, sino que estos procesos se han puesto a punto como tratamiento a las disoluciones metálicas residuales de tratamientos comerciales o para obtener una corriente salina $\mathrm{KNO}_{3} / \mathrm{KF}$ y posterior electrodiálisis ${ }^{[5]}$.

En el presente trabajo se plantea un procedimiento de precipitación selectiva de hierro y cromo en una forma que sea aprovechable por la industria. Se produce un efluente líquido en el que permanece el níquel disuelto que se conduce a una posterior etapa de precipitación. El mecanismo de precipitación que se ha propuesto es el siguiente ${ }^{[10]}$ :

$$
\begin{gathered}
\mathrm{FeF}_{2}^{-}+\mathrm{HF}+\mathrm{KOH} \rightarrow \mathrm{K}_{2} \mathrm{FeF}_{5} \cdot \mathrm{H}_{2} \mathrm{O}(\mathrm{pH}=3-4,5)(1) \\
\mathrm{K}_{2} \mathrm{FeF}_{5} \cdot \mathrm{H}_{2} \mathrm{O}+\underset{(\mathrm{pH}>10)}{\mathrm{KOH} \text { exc. } \rightarrow} \rightarrow \mathrm{Fe}(\mathrm{OH})_{3}+\mathrm{KF}
\end{gathered}
$$

En la primera etapa se desarrolla la reacción (1). El hierro y el cromo precipitan, mientras el níquel permanece en disolución, La suspensión se filtra y la corriente líquida se somete a una segunda precipitación para obtener $\mathrm{Ni}(\mathrm{OH})_{2}$. El sólido previo de pentafluoruos se hidroliza a $\mathrm{pH} 10$, precipitando los metales como hidróxido.

\section{MATERIALES Y PROCEDIMIENTO EXPERIMEN- TAL}

La instalación experimental se puede observar en la figura 1 .

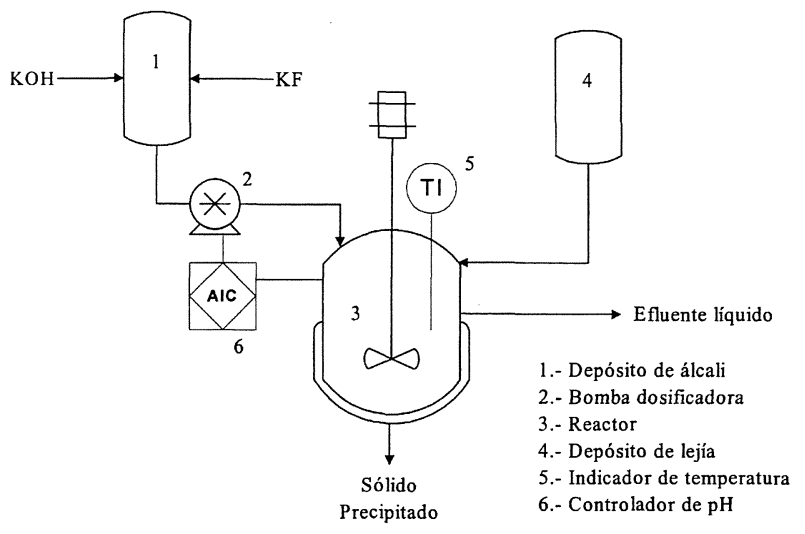

Figura 1. Instalación experimental de las reacciones de precipitación.

Figure 1. Experimental installation for the precipitation reaction.

Se dispone de un reactor de 21 con rebosadero, al que se añade una cantidad inicial de agua para evitar problemas de precipitación masiva al añadir álcali. El baño ácido agotado, con la concentración expuesta en la tabla I, se añade continuamente, mientras un controlador regula el $\mathrm{pH}$ de reacción mediante adición de potasa $1,5 \mathrm{M}$ al que se ha añadido fluoruro libre en forma de KF para completar la estequiometría de la reacción 1 . El reactor se calienta a la temperatura de operación mediante inmersión en un baño termostatizado. Las variables a estudiar son el $\mathrm{pH}$, la temperatura y la cantidad de $\mathrm{KF}$ añadido. Las condiciones constantes de reacción figuran en la tabla II.

La concentración de hierro, cromo, niquel y potasio se ha determinado mediante el equipo de absorción atómica VARIAN SPECTRAA 220. La concentración de aniones se determina mediante sendos electrodos selectivos de fluoruro y nitrato. $\mathrm{El} \mathrm{pH}$ de la reacción se controla mediante un equipo CRISON pH ROCON 18. Las muestras sólidas se caracterizaron mediante fluorescencia de rayos $X$ con el equipo BRUKER S4-EXPLORER, difracción de rayos $\mathrm{X}$ con el difractómetro SIEMENS

Tabla II. Condiciones de reacción constantes

Table II. Constant experimental conditions

\begin{tabular}{lc}
\hline \multicolumn{1}{c}{ Condiciones de operación } & Valor \\
\hline Agitación & 700 r.p.m. \\
Caudal de adición de lejía agotada & $8 \mathrm{ml} / \mathrm{min}$ \\
Volumen inicial de agua & $1.000 \mathrm{ml}$ \\
Concentración de $\mathrm{KOH}$ & $1,5 \mathrm{M}$ \\
\hline
\end{tabular}

Rev. Metal. Madrid Vol. Extr. (2005) 475-478 
D500-1 y microscopía electrónica de barrido con un microscopio JEOL 6400 a $20 \mathrm{kV}$.

\section{RESULTADOS}

\subsection{Condiciones de operación}

En la Tabla III se exponen variables estudiadas en cada ensayo realizado.

Para evaluar estos experimentos se considera el grado de separación del níquel y la cantidad que se recupera en el efluente líquido respecto de la inicial alimentada con la lejía de decapado. Estas variables son \% pureza (respecto del total metálico $\mathrm{Fe}+\mathrm{Cr}+\mathrm{Ni}$ ) y \% recuperación. Una síntesis de los resultados obtenidos se observa en la figura 2.

\subsection{Caracterización del sólido}

Se caracteriza el sólido obtenido en el experimento 7 mediante técnicas de difracción de rayos $\mathrm{X}$, fluorescencia y microscopía electrónica junto con un análisis semicuantitativo de dispersión de rayos

Tabla III. Experimentos realizados

Table III. Experimental tests

\begin{tabular}{cccc}
\hline Ensayo & $\mathbf{p H}$ & $\mathbf{T}\left({ }^{\circ} \mathrm{C}\right)$ & $\mathbf{K F}(\%$ en álcali) \\
\hline 1 & 3,5 & Ambiente & 5 \\
2 & 3,5 & 65 & 5 \\
3 & 3,5 & 65 & 10 \\
4 & 4 & 65 & 10 \\
5 & 4,5 & 65 & 10 \\
6 & 4 & 65 & 12,5 \\
7 & 4 & 65 & 15 \\
\hline
\end{tabular}

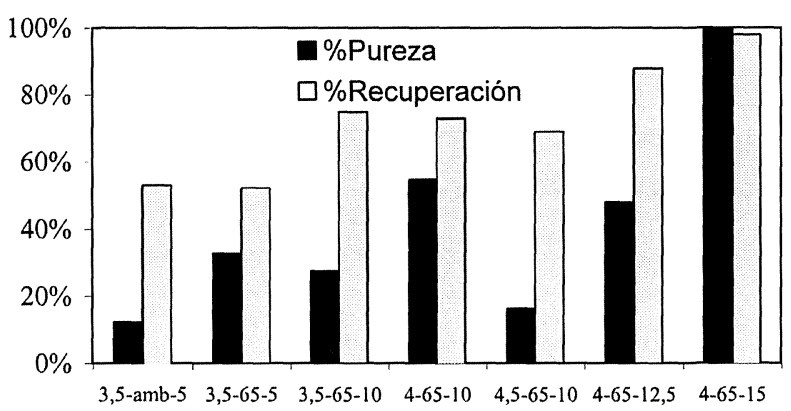

Figura 2. Recuperación y pureza del níquel en los ensayos realizados.

Figure 2. Nickel recovery and purity obtained in the experimental tests.

Rev. Metal. Madrid Vol. Extr. (2005) 475-478
$\mathrm{X}$. El análisis por difracción de rayos X demuestra que los compuestos mayoritarios son pentafluoruros de hierro y cromo hidratados con una molécula de agua, con una pequeña cantidad de hexafluoruros de ambos metales. En cuanto a la composición por elemento se realiza un estudio por balance de materia y se compara con dispersión y fluorescencia de rayos X (Tabla IV).

En la figura 3 se observa la micrografía realizada por microscopía electrónica del sólido obtenido en la precipitación del ensayo 7. Se puede observar el sistema de cristalización monoclínico centrado en la base del pentafluoruro hidratado de hierro (que es el compuesto mayoritario).

\section{DISCUSIÓN}

Según se puede observar en la figura 2, los ensayos 1 y 2 reflejan que el aumento de temperatura permite una mayor pureza de níquel en disolución porque la precipitación de hierro y cromo se ve

Tabla IV. Composición del sólido según diferentes técnicas Table IV. Solid composition measured by material balance, EDX and FRX

\begin{tabular}{lccr}
\hline & Balance & EDX & FRX \\
\hline $\mathrm{Fe}$ & $18,2 \%$ & $22,2 \%$ & $18,1 \%$ \\
$\mathrm{Cr}$ & $3,8 \%$ & $4,8 \%$ & $5,2 \%$ \\
$\mathrm{Ni}$ & $0,3 \%$ & $0 \%$ & $0,0 \%$ \\
$\mathrm{~F}$ & $37,3 \%$ & $44,4 \%$ & $40,6 \%$ \\
$\mathrm{~K}$ & $40,4 \%$ & $28,6 \%$ & $36,0 \%$ \\
\hline
\end{tabular}

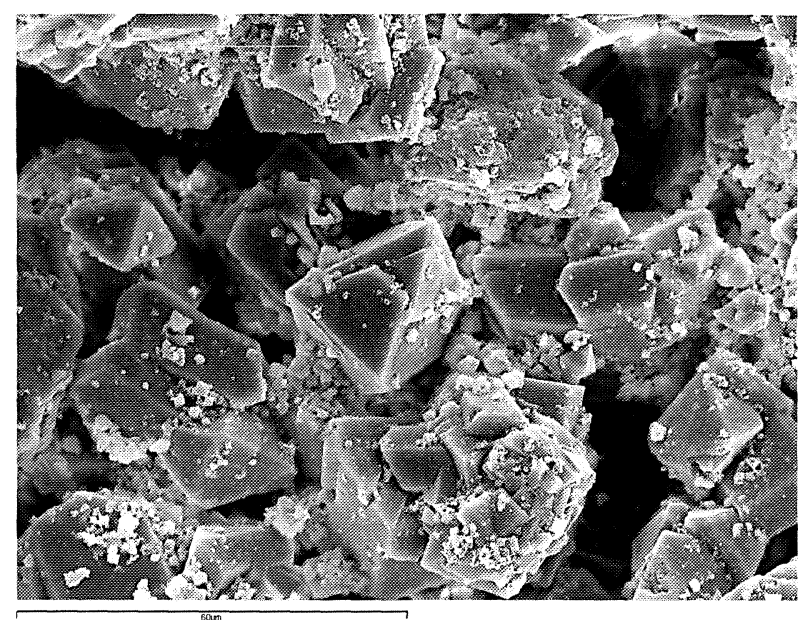

Figura 3. Micrografía del sólido obtenido en el ensayo 7.

Figure 3. Micrograph of the solid obtained in run 7. 
favorecida por la temperatura. El aumento de $\mathrm{pH}$ (ensayos 3, 4 y 5 ) no favorece la precipitación de hierro y cromo a pentafluoruro, sino que existe un $\mathrm{pH}$ óptimo para la precipitación que se sitúa a $\mathrm{pH}$ 4 , ya que la pureza disminuye considerablemente a $\mathrm{pH} 4,5$. Esto puede ser debido a la redisolución parcial de los compuestos fluorados y a que el aumento de caudal de álcali provoca menor tiempo de residencia dentro del reactor y un descenso de la concentración de fluoruro libre en el interior.

Para el ajuste del fluoruro potásico adicionado para completar la estequiometría de la reacción (1), se aumenta la concentración de KF (ensayos 4, 6 y 7). En el experimento 7, de mayor dosificación de KF (15\%), se alcanza una recuperación del $99 \%$ de níquel y una precipitación total del hierro y el cromo como pentafluoruros hidratados. Asimismo, la pureza del níquel se ve ostensiblemente mejorada (superior al 99,9\%). Este fenómeno se debe posiblemente a que la formación de los fluoruros complejos evita la coprecipitación de níquel.

Con los resultados anteriores, los valores óptimos de operación son $\mathrm{pH} 4, \mathrm{~T}=65^{\circ} \mathrm{C}$ y $15 \%$ de KF en álcali.

Otro efecto a resaltar es que no se observa incremento significativo de la concentración de fluoruro en el efluente líquido al aumentar la concentración de fluoruro potásico alimentado con el álcali, debido a que el mayor contenido adicionado reacciona con el hierro y el cromo.

En cuanto a la caracterización del sólido, la diferencia entre las distintas técnicas, observada en la tabla IV, se debe al margen de error de las técnicas semicuantitativas de fluorescencia y dispersión de rayos X. Las relaciones entre los diferentes compuestos se observan correspondientes a la estequiometría de pentafluoruros de hierro y cromo. Esto se ve confirmado por la difracción de rayos X, ya que el espectro obtenido muestra que el compuesto en mayor proporción es $\mathrm{K}_{2} \mathrm{FeF}_{5} \cdot \mathrm{H}_{2} \mathrm{O}$, al que probablemente acompaña $\mathrm{K}_{2} \mathrm{CrF}_{5}$ y, en muy poca proporción, hexafluoruros complejos de los dos metales. No aparece ninguna sal cristalizada a partir de los aniones, como $\mathrm{KF}, \mathrm{KNO}_{3}$ o $\mathrm{KOH}$.

\section{CONCLUSIONES}

Mediante una precipitación selectiva inducida por fluoruro libre se consigue separar el níquel del resto de metales presentes en un baño ácido nítrico/fluorhídrico agotado del decapado de acero inoxidable. Esta precipitación se consigue a condiciones de operación de $\mathrm{pH} 4$, temperatura de 65 ${ }^{\circ} \mathrm{C}$ y con un $15 \%$ de KF en la corriente de álcai encargada de mantener el $\mathrm{pH}$. La corriente líquida contiene más del 99 \% del níquel inicial mientras que el sólido está formado por pentafluoruos de hierro y cromo con, únicamente, trazas de nitrógeno.

\section{Agradecimientos}

Los autores desean expresar su agradecimiento por la financiación recibida al Ministerio de Ciencia y Tecnología a través del proyecto de investigación MAT2002-03435.

\section{REFERENCIAS}

[1] F. LANCELOT, Metall. Trans. 21B (1990) 63-74.

[2] C.J. Brown, Iron Steel Eng. 67 (1990) 55-60.

[3] F. Heras, Tesis Doctoral, Facultad de Ciencias Químicas, Universidad Complutense de Madrid.

[4] Y. Kobuchi, Y. Matsunaga y Y. Noma, J. Membr. Sci. 27 (1986) 173-179.

[5] J.C. Mccardle, J.A. Piccari y C.G. Thornburg, Iron Steel Eng. 68 (1991) 39-43.

[6] W. Karner, D. Wurmbauer, H. Krivanec y J. Horn, Patente Europea n ${ }^{\circ}$ ES 2022720.

[7] F.J. Alguacil, Rev. Metal. Madrid 38 (2002) 205-209.

[8] F.J. Alguacil y M. Alonso, Rev. Metal. Madrid 38 (2002) 263-269.

[9] S. OHya y S. NAKashima, Iron Control Disposal Proc. Int. Symp. Iron Control Hydrometall., $2^{\text {nd }}$ edition, 1997, pp. 169-175.

[10] J. Dufour, F. Heras, C. Negro y F. López-Mateos, ISiJ Int. 41 (2002) 801-806. 